\title{
Influence of the pulse duration at near-infrared wavelengths on the laser-induced material removal of hot-dipped galvanized steel
}

\author{
H. Mustafa, ${ }^{1, *}$ D.T.A. Matthews ${ }^{1,2}$ and G.R.B.E. Römer ${ }^{1}$ \\ ${ }^{1}$ Chair of Laser Processing, Department of Mechanics of Solids, \\ Surfaces 85 Systems $\left(\mathrm{MS}^{3}\right)$, Faculty of Engineering Technology, \\ University of Twente, Enschede, the Netherlands \\ ${ }^{2}$ Chair of Skin Tribology, Department of Mechanics of Solids, \\ Surfaces 85 Systems $\left(\mathrm{MS}^{3}\right)$, University of Twente, Enschede, the Netherlands
}

(Dated: March 25, 2020)

\begin{abstract}
Hot-dipped galvanized steel is processed with short and ultrashort pulsed lasers in air at near infra-red wavelengths with pulse durations ranging from 350 fs to $241 \mathrm{~ns}$. The morphology of the ablated craters (processed over a range of laser fluence levels and number of laser pulses) are analyzed by confocal laser scanning microscopy and scanning electron microscopy. The ablation threshold of galvanized steel is found to increase with laser pulse durations following a simple power $F_{t h}=A \cdot \tau^{B}$ law. Longer pulse durations in the ns regime, as compared to pulse durations in ps and fs regime, results in higher ablation efficiency and energy penetration depth at the cost of surface quality.
\end{abstract}

Keywords: Ultrashort pulsed laser, galvanized steel, ablation threshold, ablation efficiency, rim

\footnotetext{
*h.mustafa@utwente.nl
} 


\section{INTRODUCTION}

Surface texturing offers ways to improve the functionality of a surface [1]. Short and ultrashort pulsed lasers are used extensively for this purpose, since they offer accurate removal of material [2]. The duration of each laser pulse determines how long the intense laser pulse interacts with the target material. Consequently, different surface morphologies result from the laser-material interaction, when applying different pulse durations. Furthermore, the processing quality and the material removal rate also depend on the laser pulse duration [36]. In order to use (ultra)short-pulsed lasers for the purpose of surface texturing, knowledge of material response at different laser pulse durations is one of the fundamental requirements.

Material removal due to laser-material interactions can be considered in three stages that involve both the target sample and the ablated particles, namely, (a) laser absorption, subsequent heating [7] and plasma generation [8], (b) plasma expansion [8] and cooling of the sample [7] as well as of the plasma [8] and, (c) solidification of nonablated surface [7] and condensation of the ablated particles [8]. While the latter two stages typically take place after the laser pulse, the temporal width of the laser pulse $\left(\tau_{L}\right)$ determines primarily the heating of the target sample and possible interaction of the laser beam with the ablated particles, e.g. plasma shielding. The thermalization of the irradiated sample happens within a volume given by the laser spot diameter and the optical penetration depth $\delta_{\text {opt }}=1 / \alpha$, where $\alpha$ is the attenuation coefficient of the target material at a given laser wavelength [2]. For $\tau_{L}$ shorter than the characteristic electron-phonon coupling time $\tau_{e-p h}$, the thermalization of the irradiated sample follows a two-temperature model (TTM) involving the temperature of the electronic $\left(T_{e}\right)$ and lattice subsystem $\left(T_{l}\right)[2,9-12]$. Within the aforementioned interacting volume, the laser energy is absorbed by the free electrons in metal by inverse Bremsstrahlung, perturbs the fermi distribution of the electrons and creates ballistic electrons $[9,13]$. The free carrier absorption in metal leads to thermalization of the electronic subsystem on a timescale approximately equal to the plasma frequency $\left(\omega_{p}^{-1}\right)[14]$. For femtosecond laser pulses, the laser excited electrons reach a very high temperature while the lattice remains cold. As a consequence, the lattice experiences a strong force due to the changes in potential energy surface and disorders like 'melting' long before the electron-phonon heating [13]. This phenomenon is known as nonthermal melting. Furthermore, the laser excited electrons, 
also known as ballistic electrons, penetrate within the material at their mean free path [13]. Therefore, the effective energy penetration depth includes both the optical and the ballistic electron penetration depth [15]. Thermalized electrons, resulting from the electron-electron collision, relax with the initially cold lattice through electron-phonon collisions. Within a characteristic coupling time $\tau_{e-p h}$, the relaxation of the electronic and the lattice subsystem leads to a joint temperature, i.e. the lattice temperature increases [10]. At low laser fluences close to the melting threshold, laser irradiation results in fast melting followed by the epitaxial regrowth of the crystalline bulk and complete resolidification [16]. At higher fluences, melting kinetics become a combination of fast homogeneous melting followed by slower heterogeneous melting at the liquid-crystal interface [7]. If $\tau_{L}$ is greater than $\tau_{e-p h}$, there lies no disparity between the electron and lattice temperature within the duration of the laser pulse. In this case, heat penetrates from the surface to the bulk following classical heat transport equation within a heat penetration depth $\delta_{h}=2 \cdot \sqrt{D \cdot \tau_{L}}$, where $D$ is the heat diffusion coefficient [2]. For short laser pulses with sufficient intensity, the irradiated material undergoes rapid, isochoric heating. If the sample is overheated beyond the thermodynamic stability (close to the critical temperature $T_{c}$ ), the material decomposes in an explosive manner, known as explosive boiling or phase explosion [7, 12]. In such cases, pulse durations shorter than the thermal diffusion time results in thermal confinement of the absorbed laser energy $[17,18]$. On the other hand, pulse durations shorter than the transit time of the sound wave within the irradiated volume results in stress confinement $[17,18]$. In this case, a strong compressive stress is built up at the target surface, resulting in photomechanical spallation [12]. These photomechanical and photothermal processes result in ejection of single and/or multiple atomic layers as well as large liquid droplets and vapor atoms. These ablated particles along with the particles in the ambient environment make up the ablated plume/plasma [8]. If $\tau_{L}$ is longer than the plasma formation time, part of the laser beam is scattered, reflected and absorbed by the plasma particles through inverse Bremsstrahlung (IB) and photoionization (PI) processes $[2,8,19]$. As a consequence, the available laser energy on the target sample reduces, and the phenomenon is known as plasma shielding. After the laser pulse, the expanding plume/plasma above the Knudsen layer exerts a recoil pressure over the molten surface and expels the melt radially towards the edge [20]. The cooling of the nonablated molten material takes place through thermal diffusion in to the bulk $[7,16]$. Depending on the lifetime and depth of the melt, the nonablated 
molten material can quench at a very high rate [21]. Rapid solidification of the nonablated material results in hydrodynamic instabilities in the melt flow [22], and affects the nucleation process $[7,21]$. The condensation of the ablated particles leads to the redeposition of micro- and nanoparticles in and around the laser irradiated zone $[2,8]$. Therefore, the final morphology of the laser irradiated zone largely depends on the duration of the laser pulse due to the complex nature of laser ablation. Since the material of interest in this work is galvanized steel, the initial interactions of the laser beam with the material happens on the zinc coating. Interestingly, the morphology of the laser irradiated zinc surface is always associated with the presence of melt, regardless of femto [23], pico [24] or nanosecond [25] pulse duration. In Fig. 1, some of the material dependent timescales are shown for zinc along with the investigated pulse durations in this work. The associated equations and material properties can be found in the appendix. Together, these timescales determine the evolved surface morphology for processing with multiple pulses at different fluence levels.

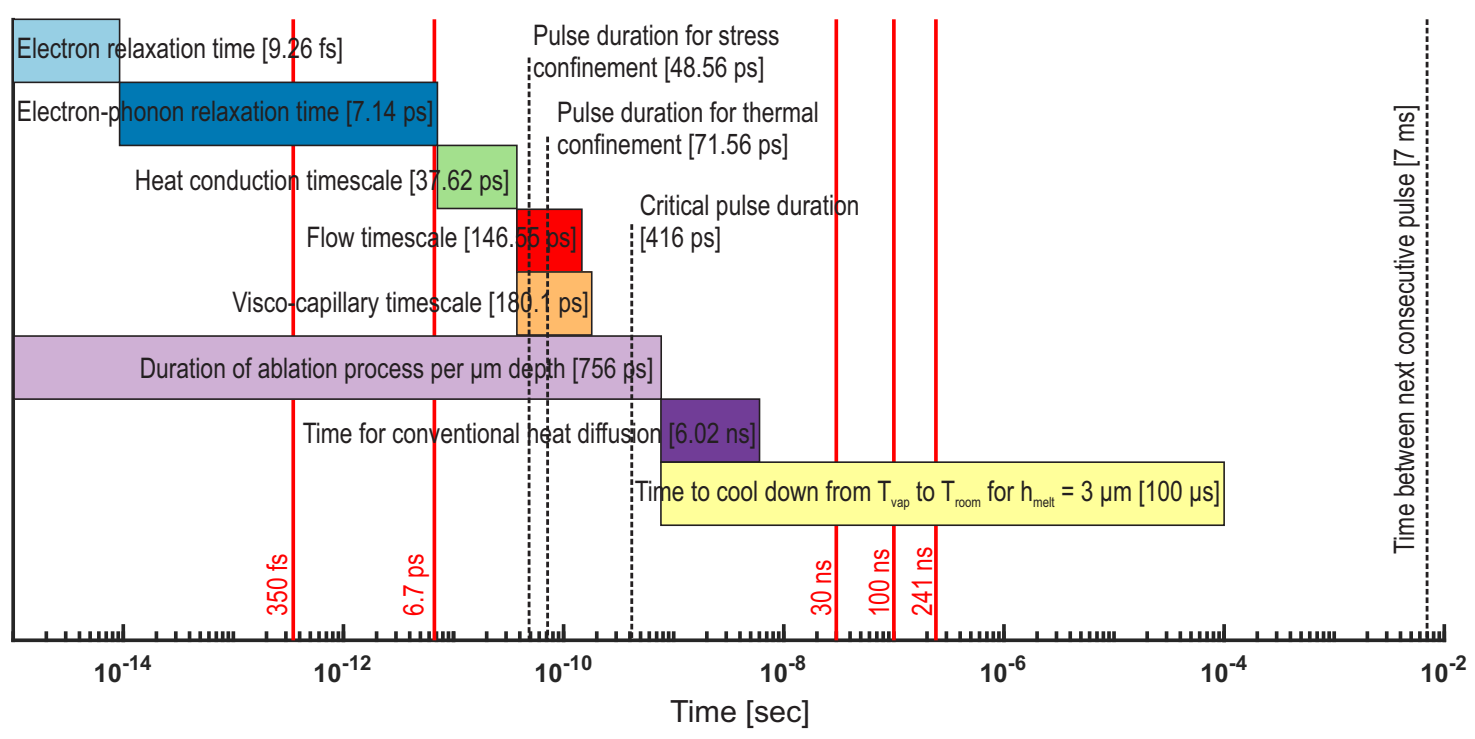

FIG. 1. Theoretical timescales of different physical mechanisms associated with laser ablation of Zn. The vertical red solid lines indicate pulse duration of the laser sources used in this work, whereas the vertical black dashed lines indicate limitations on pulse durations with different mechanisms.

Zinc is one of the most used metals and almost half of it finds its use in galvanizing [26]. Galvanized steel (GI) is extensively used in transportation, construction, chemical, oil and gas industries among others [27]. Galvanized steel is typically processed with (ultra)short 
pulsed lasers for characterizing its coating thickness using laser induced breakdown spectroscopy (LIBS) [28-35]. Studies of depth profiling using single [29], double [30] and multiple [28] laser pulses to achieve high depth resolution and signal sensitivity for Zn-coated steel have been reported. However, the surface chemical composition, microstructures and surface roughness of Zn-coated steel are susceptible to the Zn deposition process during galvanizing $[36,37]$. Therefore, not all reported literature on LIBS can be directly correlated for surface texturing. The improvement of functionality of the zinc coating on galvanized steel has been performed either chemically and/or mechanically [38-40]. Laser surface texturing has the potential to enable further improvements in mainstream functional properties involving tribology (roughness, friction) [41], optics (visual appearance) [42] or adhesion (paint, glue, lubricant) [43].

In this work, laser ablation of hot-dipped galvanized steel is presented at near IR wavelengths from 350 fs to $241 \mathrm{~ns}$ in order to investigate the influence of laser pulse duration on the laser induced material removal with a three-fold purpose. Firstly, the laser irradiated surface morphologies are analyzed to describe the surface structures and to identify possible physical phenomena occurring. Secondly, the dependence of fluence ablation threshold and ablation efficiency on laser pulse duration is investigated for single and multiple laser pulse irradiation. Thirdly, laser micromachining using different laser pulse duration as a surface texturing process is evaluated in terms of depth resolution and processed surface quality.

\section{EXPERIMENTAL}

\section{A. Laser setup}

The ablation experiments were performed under atmospheric conditions in a cleanroom environment using three different laser sources, see Table I. All the laser sources emit at near infra-red wavelengths and show a nearly Gaussian power density profile $\left(M^{2}<1.3\right)$. A galvo-scanner (IntelliScan14 of ScanLab GmbH, Germany), equipped with a telecentric flatfield F-theta-Ronar lens (Linos GmbH, Germany) was used to scan the focused laser beam over the surface of the sample. The sample was placed in the focal plane. The focal spot radius was measured from the fluence profile using a charge-coupled device (CCD) 
TABLE I. Laser sources used in this work.

\begin{tabular}{|c|c|c|c|c|c|}
\hline Parameter & & $\begin{array}{c}\text { SPI } \\
\text { edEnergy G }\end{array}$ & & $\begin{array}{c}\text { Trumpf } \\
\text { TruMicro } 5050\end{array}$ & $\begin{array}{c}\text { KMLabs } \\
\text { Y-Fi }\end{array}$ \\
\hline Wavelength $[\mathrm{nm}]$ & & 1060 & & 1030 & 1040 \\
\hline Output power instability [\%] & & $<5$ & & $<2$ & $<2$ \\
\hline Pulse duration & $241 \mathrm{~ns}$ & $100 \mathrm{~ns}$ & $30 \mathrm{~ns}$ & $6.7 \mathrm{ps}$ & $350 \mathrm{fs}$ \\
\hline Focal spot radius $[\mu \mathrm{m}]$ & $15.2 \pm 1.6$ & $14.6 \pm 1.6$ & $14.2 \pm 1.6$ & $14.4 \pm 1.6$ & $9.6 \pm 1.6$ \\
\hline Ellipticity of focal spot & 0.83 & 0.82 & 0.81 & 0.9 & 0.67 \\
\hline Rayleigh range $[\mathrm{mm}]$ & 0.77 & 0.58 & 0.59 & 0.56 & 0.28 \\
\hline Time between consecutive pulses [ms] & $<7.1$ & $<7.1$ & $<7.1$ & $<7.1$ & 0.001 \\
\hline Number of pulses & $1-50$ & $1-50$ & $1-50$ & $1-50$ & $50 \cdot 10^{3}-1 \cdot 10^{6}$ \\
\hline Pulse energy $[\mu \mathrm{J}]$ & $78.5-260$ & $60.8-152.5$ & $38.5-63.6$ & $6.0-119.3$ & $0.1-0.7$ \\
\hline
\end{tabular}

sensor-based, beam diagnostic system (MicroSpot Monitor of Primes GmbH, Germany). The pulse width of femto- and picosecond laser sources were verified using an autocorrelator (PulseCheck of Angewandte Physik \& Elektronik GmbH, Germany). The pulse width from the autocorrelation function (ACF) was calculated by multiplying the full width at half maximum (FWHM) of ACF with a Gaussian form factor (0.71). For nanosecond laser sources, pulse width verification were performed using a high-speed Si photodiode (DET10A/M of Thorlabs, USA) in combination with a GHz oscilloscope (LeCroy WavePro 7000A of Teledyne Technologies Inc., USA). In this case, duration of a pulse was considered at $10 \%$ from the photodiode signal peak. The laser beam impinges perpendicular to the sample surface. The focus position was fixed for all the experiments and coincided with the original surface. An exhaust system was used to extract airborne debris from the laser-material interaction zone during processing. The laser energy supplied to the surface was varied by using a combination of a half-wave plate and a polarizing beam splitter. A pyroelectric detector (PM30 with FieldMax II of Coherent, USA) was used to measure the average laser power incident at the sample with an error less than $8 \%$. The energy of the individual pulses was determined by dividing the measured average laser power by the pulse frequency applied. The geometrical pulse-to-pulse distance was at least $125 \mu \mathrm{m}$. The number of pulses on the same location varied from $N=1$ to 50 for the ps and ns laser sources. At a repetition rate of $8 \mathrm{kHz}$ and a beam scanning velocity of $1 \mathrm{~m} / \mathrm{s}$, time between consecutive pulses on 
the same location equals at least $7 \mathrm{~ms}$. For the fs laser source, the number of pulses varied from $N=50 \cdot 10^{3}$ to $1 \cdot 10^{6}$ and the time between consecutive pulses equals $1 \mu \mathrm{s}$. A total of 30 craters were created per laser setting to ensure statistically sound values in measured quantities.

\section{B. Material}

Galvanized steel, having a Zinc coating $\left(99.7 \% \mathrm{Zn}, 0.3 \% \mathrm{Al}\right.$ ) weight of $70 \mathrm{~g} / \mathrm{m}^{2}$ (average thickness of $8 \pm 2 \mu \mathrm{m}$ ), deposited by an industrial, continuous Hot Dip Galvanizing (HDG) on Titanium Stabilized Ultra Low Carbon (TiSULC) steel substrate, was used. The samples are commercially produced according to European standard EN10346:2015 with a surface roughness $\left(R_{a}\right)$ of $0.5 \mu \mathrm{m}$. Samples were cleaned using Ammonia $(<5 \%)$ solution $\left(\mathrm{NH}_{3}(a q)\right)$ prior to and after the laser ablation experiments.

\section{Analysis Tools}

The laser-induced surface profiles (the latter referred to as 'craters 'in the remainder of this paper), were measured by means of Confocal Laser Scanning Microscopy (CLSM), (VK-9700 of Keyence Corporation, Japan). The lateral and vertical resolutions of CLSM measurements were $276 \mathrm{~nm}$ and $1 \mathrm{~nm}$ respectively. Laser-induced crater morphology was analyzed by means of a field emission Scanning Electron Microscope (SEM), (JSM-7200F of Jeol, Japan).

\section{RESULTS}

To identify the effect of laser pulse duration on material removal, first the morphology of the craters, processed at different pulse durations with different numbers of pulses and different fluence levels, are investigated in Sec. III A. Next, the ablation thresholds and ablation efficiency are discussed in Sec. III B. 


\section{A. Crater morphology}

Experiments at femtosecond pulse duration were performed at lower fluence levels $\left(F_{0}\right)$ as well as at a higher number of laser pulses $(N)$ than the experiments performed at picoand nanosecond pulse durations. Therefore, a direct comparison in terms of $F_{0}$ and $N$ is performed for picosecond and nanosecond pulse durations in this subsection. In Fig. 2, CLSM images of craters processed at $F_{0}=20 \mathrm{~J} / \mathrm{cm}^{2}$ with $N=1$ and 10 are shown for laser pulse durations of $6.7 \mathrm{ps}, 30 \mathrm{~ns}, 100 \mathrm{~ns}$ and $241 \mathrm{~ns}$. In these images, the laser beam

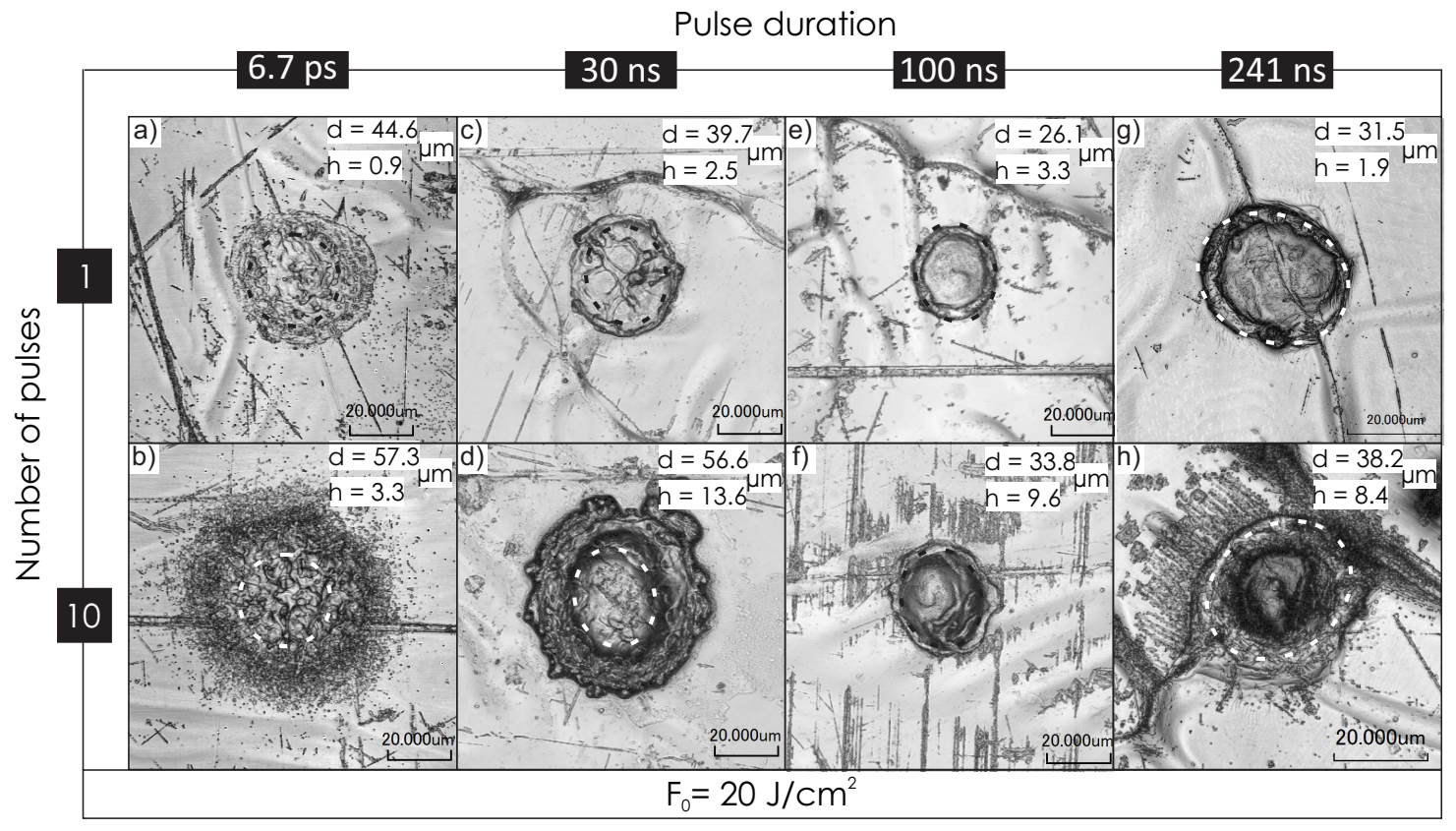

FIG. 2. Confocal laser scanning microscope images of galvanized steel surface irradiated with laser pulses of different pulse durations at $F_{0}=20 \mathrm{~J} / \mathrm{cm}^{2}$ for $N=1$ and 10 . The dotted black circle indicates the laser beam diameter $\left(1 / e^{2}\right)$. Diameter, $d$ and maximum depth, $h$ of the modified surface are derived from CLSM measurements. All images are in same scale.

diameter at $1 / e^{2}$ is shown by the dotted circle. As can be observed, surface modification is not limited within the the $1 / e^{2}$ laser beam diameter. This observation is more apparent for shorter pulse durations than the longer ones (see also Fig. 3).

For longer laser pulses (see Fig. 2(e) and $(\mathrm{g})$ ), the crater diameter is well within the $1 / e^{2}$ laser beam diameter. These craters show a smooth central modification zone followed by a well-defined rim at the edge of the crater. On the other hand, at a pulse duration of $30 \mathrm{~ns}$ (see Fig. 2(c)), the central modified zone of the crater shows melt instabilities in the form 


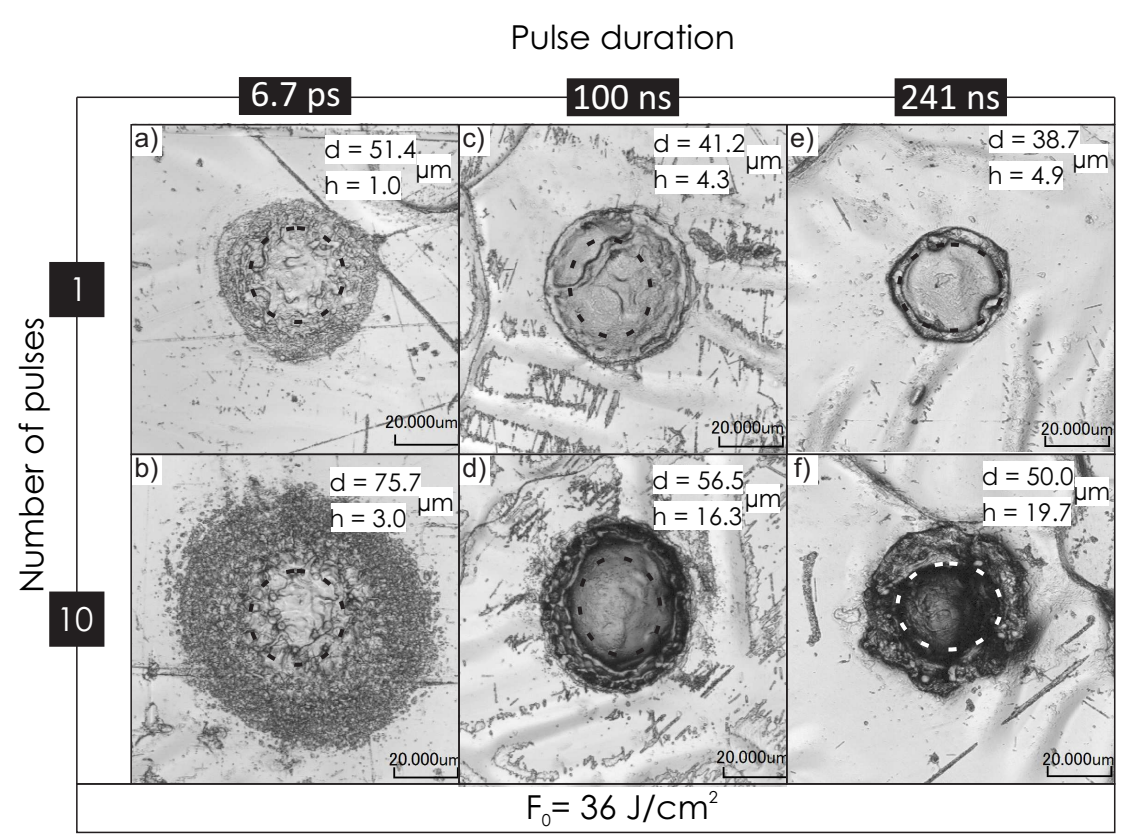

FIG. 3. Confocal laser scanning microscope images of galvanized steel surface irradiated with laser pulses of different pulse durations at $F_{0}=36 \mathrm{~J} / \mathrm{cm}^{2}$ for $N=1$ and 10 . The dashed circle indicates the laser beam diameter $\left(1 / e^{2}\right)$. Diameter, $d$ and maximum depth, $h$ of the modified surface are derived from CLSM measurements. All images are in same scale.

of protrusions. In this case, the rim around the crater forms outside the $1 / e^{2}$ laser beam diameter. The region of surface modification outside the $1 / e^{2}$ laser beam diameter increases for an even shorter pulse duration (6.7 ps), in which case, the center of the crater exhibits significant melt instabilities (see Fig. 2(a)). As can be seen from the inset texts, the crater diameter decreases and the depth of the craters increases with increasing pulse duration for a single laser pulse $(N=1)$ (see Fig. 2(a),(c),(e) and (g)). Analogously, the central part of the craters, in these figures, gradually develops from "splashes with crowns" [44] towards a "splash with primary vortex ring" [44] for $N=1$. This observation indicates that (a) shorter solidification time for shorter laser pulses results in "frozen" melt instabilities like protrusions, (b) the intensity of the tails of the Gaussian beam becomes high enough to cause material modification in case of (ultra) short(er) pulses [22, 45], and (c) the recoil pressure from the ablated plume expels the molten material towards the edge of the crater for longer pulses. With multiple pulses $(N=10)$, the mesoscale surface modification outside the crater becomes more prominent for ps laser pulses (see Fig. 2(b)). On the other hand, the height of the rim around the crater increases when processed with multiple ns laser 
pulses (see Fig. $2(\mathrm{~d}),(\mathrm{f}),(\mathrm{h}))$. At $N=10$, the redeposited particles around the crater for ps pulses might result from the Rayleigh-Plateau instability of the accelerating crowns (see Fig. 2(d)), whereas the stacking of primary vortex rings around the crater results in a well defined rim (see Fig. 2 (d),(f),(h)).

These observations are further confirmed at a higher fluence level. In Fig. 3 craters processed at $F_{0}=36 \mathrm{~J} / \mathrm{cm}^{2}$ are shown for laser pulse duration of $6.7 \mathrm{ps}, 100$ ns and 241 ns. With decreasing pulse duration, the intensity of the laser pulse increases. Therefore, the surface modification happens over a larger area than the $1 / e^{2}$ laser beam diameter at a pulse duration of $100 \mathrm{~ns}$ at this fluence level (see Fig. 2(c),(d)) than at $F_{0}=20 \mathrm{~J} / \mathrm{cm}^{2}$. Moreover, the ablated depth increases substantially more for nanosecond pulse durations than picosecond pulse duration at $F_{0}=36 \mathrm{~J} / \mathrm{cm}^{2}$ than at $F_{0}=20 \mathrm{~J} / \mathrm{cm}^{2}$. This observation suggests that the amount of the ablated plasma and its subsequent recoil pressure increases with increasing pulse duration at this fluence level.

As the number of laser pulses increases on the same location, the rim formation is seen to increase significantly with the increasing laser pulse duration. As shown in Fig. 4 for 50 pulses $(N=50)$, the deeper crater at $241 \mathrm{~ns}$ is associated with a rim as high as $6 \mu \mathrm{m}$, while for 6.7 ps pulses, the rim is absent. Also at $6.7 \mathrm{ps}$, the crater depth saturates, but the diameter increases. Therefore a heat affected zone is observed around the crater.
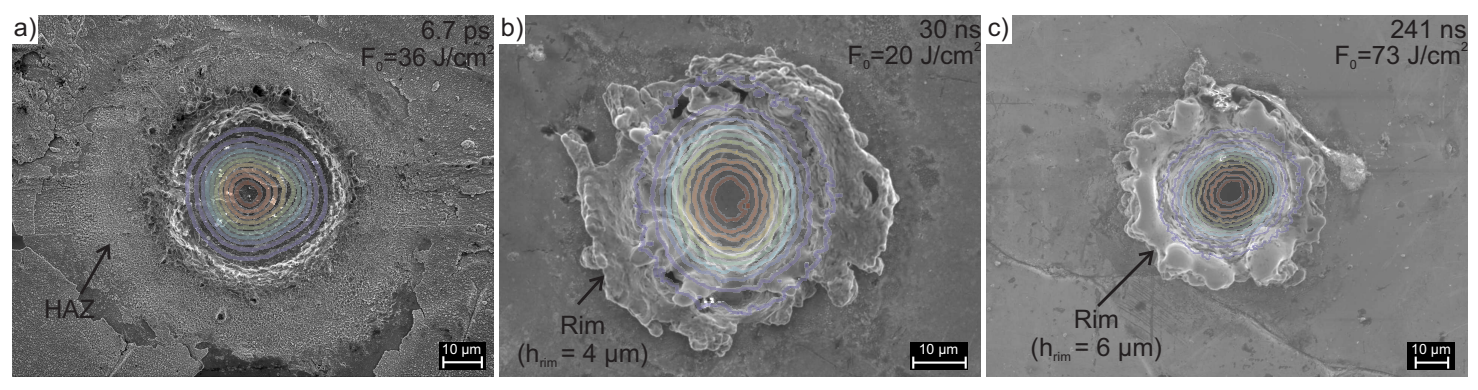

FIG. 4. Scanning electron microscopy images of laser processed galvanized steel surface irradiated with 50 laser pulses having a pulse duration of (a) $6.7 \mathrm{ps,} \mathrm{(b)} 30 \mathrm{~ns}$ and (c) 241 ns. The contour plots of corresponding laser beam intensity profile is overlaid over the craters.

The increase in geometrical dimensions (diameter, depth and volume) of the craters with 
increasing fluence and/or number of pulses is prevalent for all pulse durations. Figure 5 shows the contour plots of the ablated volume of the laser ablated craters processed at different laser pulse durations. The ablated volume increases with increasing fluence and number of laser pulses. Although saturation in depth, with increasing fluence and number of pulses, is only observed for ps laser processed samples (see Fig. 8(a)), the increasing volume indicates that the material removal happens laterally rather than vertically [6].
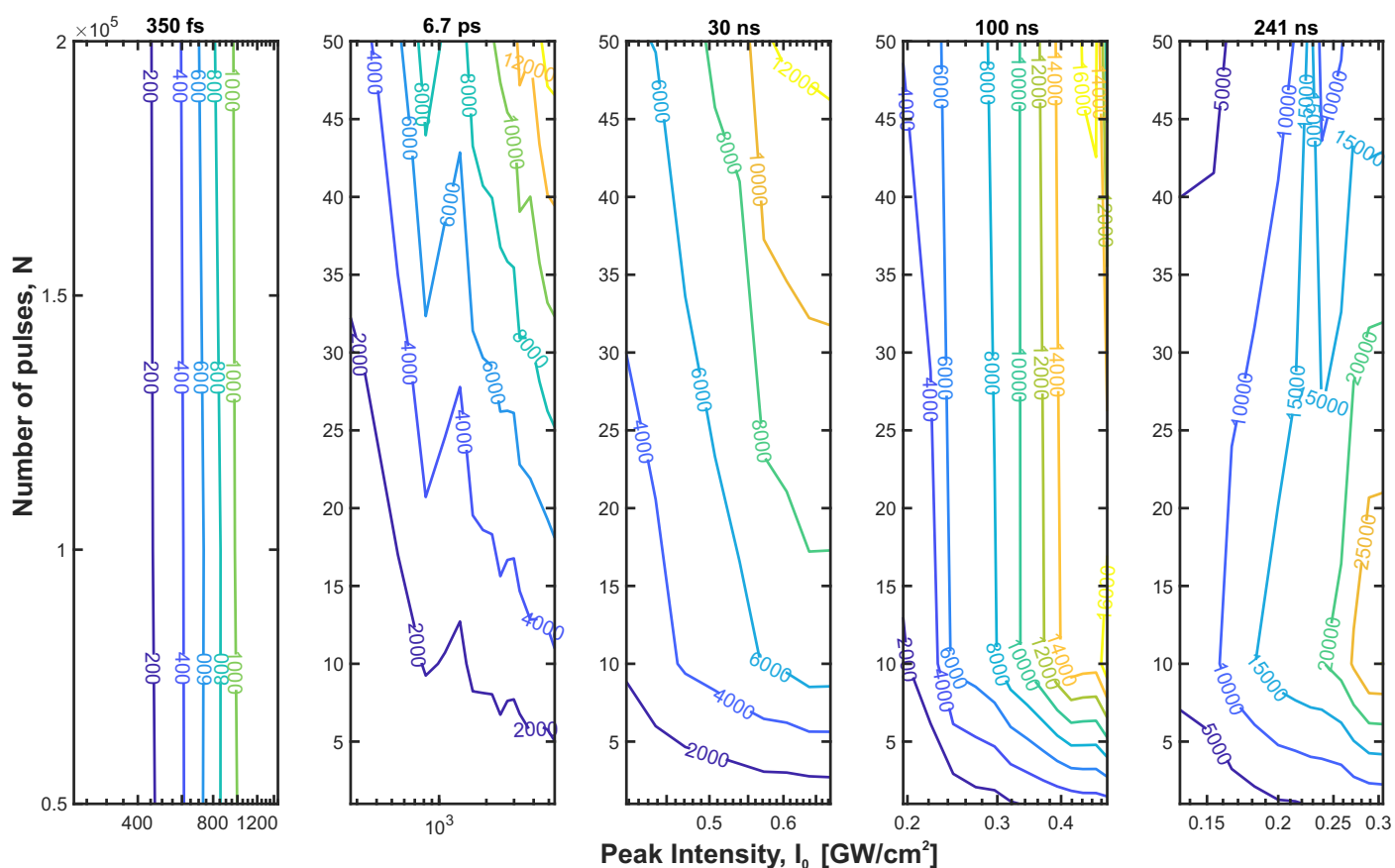

FIG. 5. Contour plots of volume of the ablated craters as a function of incident number of laser pulses $N$ and peak intensity $I_{0}$ at different pulse durations.

\section{B. Ablation threshold and ablation efficiency}

The ablation laser fluence threshold of materials can be determined using a variety of methods [5, 46-51]. Of these methods, the semilogarithmic relationship between the ablated crater diameter squared $D^{2}$ and the laser peak fluence $F_{0}$ is the most used method [47] and results in more reliable threshold determination for rough surfaces than depth or volume methods $[24,52]$. For a Gaussian fluence profile, the ablated crater diameter, $D$ and the 
peak laser fluence, $F_{0}$ is expressed as [47]

$$
D^{2}=2 \cdot \omega_{0}^{2} \cdot \ln \left(\frac{F_{0}}{F_{t h}}\right) .
$$

where, $\omega_{0}$ denotes the beam spot radius $\left(1 / e^{2}\right), F_{t h}$ is the threshold fluence and $F_{0}$ is the peak fluence, which is related to the pulse energy, $E_{p}$ as $F_{0}=\left(2 \cdot E_{p}\right) /\left(\pi \cdot \omega_{0}^{2}\right)$. In experiments, $F_{0}$ is varied and the resulting crater diameter $D$ is measured. Afterwards, $D^{2}$ is plotted against $F_{0}$ in a semilog scale and fitted with Eq. 1. The extrapolation of this curve to $D^{2}=0$ results in threshold fluence $F_{t h}$ for respective number of pulses (see Fig. 6(a)). If single pulse measurements are not possible, threshold fluence for $N=1$ can be estimated from the incubation over multiple pulses given by $N \cdot F_{t h}(N)=F_{t h}(1) \cdot N^{\zeta}$ [53]. In Fig. 6(b), the threshold fluence for $N=1$ is plotted against their corresponding pulse durations. For galvanized steel, there appears to be a power relationship between threshold fluence and pulse duration as

$$
F_{t h}=A \cdot \tau^{B}
$$

where $A=0.2 \mathrm{GW} / \mathrm{cm}^{2}$ and $B_{F_{t h}}=0.6$ are material dependent parameters. To check the extent of applicability of Eq. (2) to other material systems, the equation was fitted for $\mathrm{Cu}$ [54], stainless steel [4], barium borosilicate glass (corning 7059) [55], fused silica [56] as well as biological tissues, such as corneal stroma [56]. As can be observed from Fig. 6, this power relationship appears to be valid for any material, with a $R^{2}>80 \%$ in each case.

Within the boundaries of our experimental conditions, the ablated volume of a laser induced crater always increases with the number of laser pulses $N$ and the peak fluence $F_{0}$. The ablated volume per pulse $\Delta V$ increases as a quadratic function of the natural logarithm of peak laser fluence $F_{0}$ as $[51,57]$

$$
\Delta V=\frac{1}{4} \cdot \pi\left(\omega_{m a j} \cdot \omega_{m i n}\right) \cdot \delta_{e} \cdot\left[\ln \left(\frac{F_{0}}{F_{t h}}\right)\right]^{2} .
$$

where, $\omega_{m a j}$ and $\omega_{m i n}$ are the major and minor radius of the elliptical beam at $1 / e^{2}, \delta_{e}$ is the energy penetration depth and $F_{t h}$ is the threshold fluence. A quadratic fit according to Eq. (3) results in energy penetration depth $\delta_{e}$. The energy penetration depth $\delta_{e}$ indicates that, given the ablated volume, how deep the energy of the laser pulse has penetrated within the material. Figure 6(c) shows the ablated volume for $N=1$ for all the laser pulse durations, 

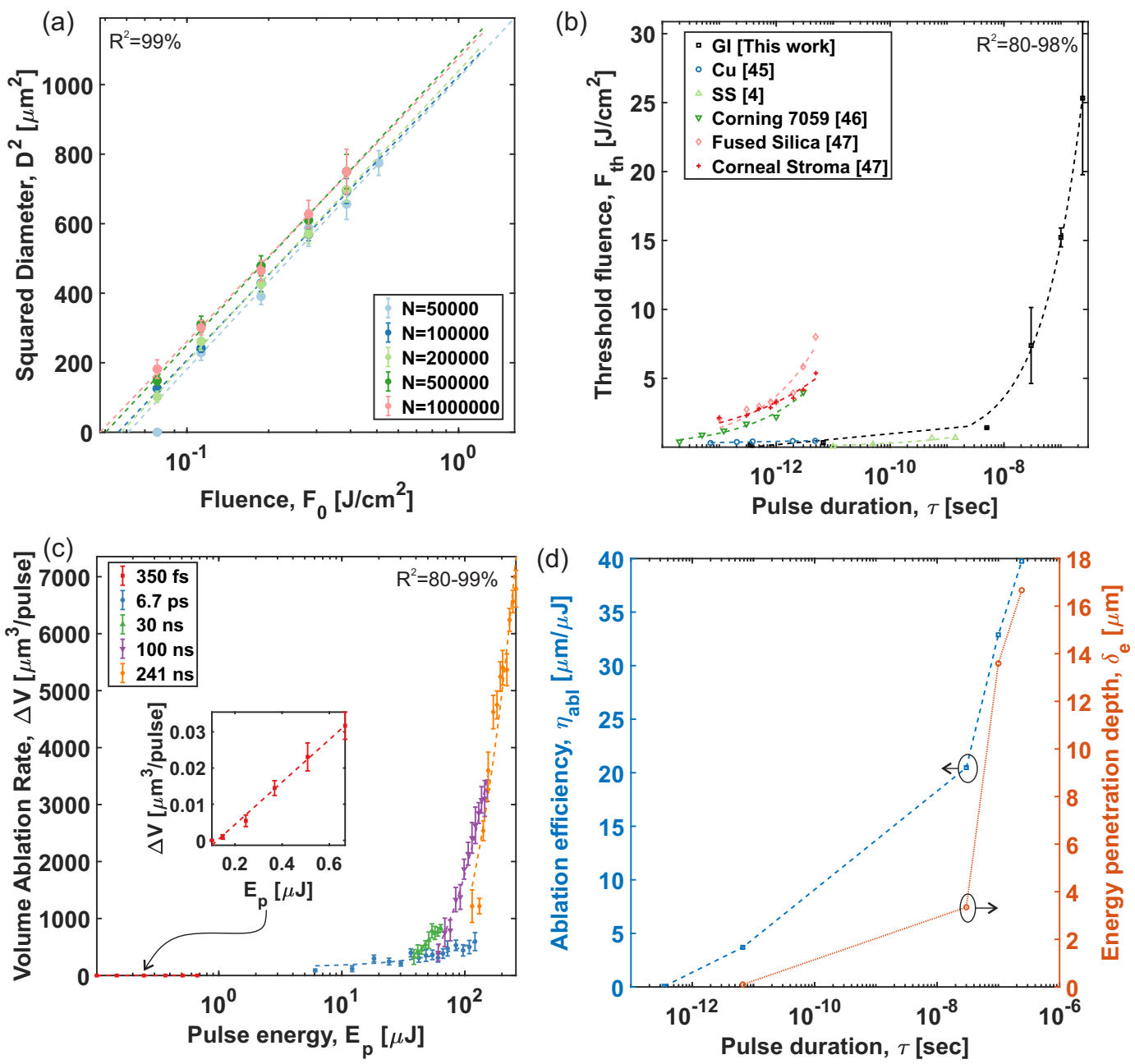

FIG. 6. (a) Squared diameter $D^{2}$ of the ablated crater for different number of pulses as a function of the peak laser fluence (log scale) at a pulse duration of $350 \mathrm{fs}$. The solid curves represent the least squared fit according to Eq. (1). (b) Dependence of ablation threshold fluence $F_{t h}$ on the laser pulse duration $\tau$ for galvanized steel. Data for $\mathrm{Cu}$, stainless steel, corning 7059 , fused silica and corneal stroma were taken from Ref. [54], [4], [55], [56] and [56], respectively. (c) Ablated volume per pulse $\Delta V$ as a function of pulse energy $E_{p}$ for $N=1$ at all laser pulse durations except $350 \mathrm{fs}\left(N=50 \times 10^{3}\right)$. The dashed lines represent linear fit through the data points. Inset shows the dependence of $\Delta V$ on $E_{p}$ for 350 fs laser pulses. (d) Dependence of ablation efficiency $\eta_{a b l}$ and energy penetration depth $\delta_{e}$ on the laser pulse duration for galvanized steel. $\delta_{e}$ was calculated from the fit of Eq. (3). 
except for 350 fs where $N=50 \cdot 10^{3}$. Clearly, the longer the duration of the pulse, the higher the ablated volume. Although the fluence of the laser pulses at longer pulse duration is higher, the slope of $\Delta V$ increases with increasing pulse duration. Typically, ablation efficiency is defined as the amount of ablated material (depth, volume, mass) for an unit of laser pulse energy or number of pulses $[6,58]$. For coated metals, such as galvanized steel, a change of material at the coating-substrate interface affects the maximum ablated depth (see Fig. 8 (a)). Since ablated volume is less affected by the coating-substrate interface, ablation efficiency $\eta_{a b l}$, in this work, is defined as ablated volume per laser pulse energy $E_{p}$. The slope of a linear fit through the data points at different pulse durations in Fig. 6(c) results in ablation efficiency $\eta_{a b l}$. In Fig. $6(\mathrm{~d}), \eta_{a b l}$ and $\delta_{e}$ is plotted against laser pulse duration. In this figure, the positive correlation between the ablation efficiency and energy penetration depth indicates that the higher $\eta_{a b l}$ at longer pulse durations results from the deeper penetration of the laser energy than the shorter pulse durations. Similar to threshold fluence, the ablation efficiency also follows a power law with an exponent value of $B_{\eta}=0.29$, which is about half of $B_{F_{t h}}$. This behavior implies that (a) the absorbed laser energy penetrates deeper within the material as laser pulse duration increases, thereby increasing the local interacting volume in the irradiated sample, (b) larger interaction volume requires higher fluence to initiate ablation, (c) larger interaction volume also increases the amount of ablated material, thereby, the ablation efficiency and, (d) the ablation efficiency of galvanized steel scales with the square root of ablation fluence threshold at a given laser pulse duration.

\section{DISCUSSION}

Heat transport is essentially a time dependent phenomenon. The interaction time of the laser pulse with the sample surface thereby determines the subsequent material response. That is, laser pulses having equal pulse energy but different pulse durations will influence the underlying mechanisms of material removal by laser ablation differently. In order to visualize this from a physical aspect, we plot the measured temporal profile of the laser pulses in Figure 7(a), where the insets show the normalized temporal profiles of femto- and picosecond laser pulses. In this figure, the energy per pulse is the area under the curve and equal in all cases. As can be observed from Fig. 7(a), the incident energy of the laser pulse 
is spread over longer time duration for the laser pulses having longer pulse duration. In Fig. $7(\mathrm{~b})$, the intensity of incident laser pulses $I_{O}$ at a constant peak fluence $F_{0}=20 \mathrm{~J} / \mathrm{cm}^{2}$ (solid curve), as well as the minimum absorbed laser intensity required to initiate stationary evaporation of the material within the duration of the laser pulse $I_{\text {evap }}$ [2] (dashed-curve) are plotted against the laser pulse duration on the left y-axis. In the same figure on the right axis, the theoretical heat penetration depth $\delta_{h}$ is plotted against the laser pulse duration. For the same amount of fluence, the intensity increases with decreasing pulse duration. On the other hand, the heat penetration depth from the surface to the bulk of the material, i.e. bulk diffusion [59], increases with increasing pulse duration. For the same absorbed energy per unit area, the slope of the thermal gradient at the vapor-surface interface becomes steeper than the thermal gradient that extends in the bulk as the pulse duration shortens [59]. That is, higher intensity results in higher surface temperature and lower bulk diffusion at a constant fluence. Consequently, threshold fluence decreases as the laser pulse duration decreases (see Fig. 6(b)).

The change of threshold fluence with laser pulse duration, expressed by Eq. (2), is similar to the well-known dependency of $F_{t h}$ on $\sqrt{\tau_{L}}[2,59]$, as well as on the $m$-photon absorption process $[46,54]$. The relation $F_{t h} \propto \sqrt{\tau_{L}}$ derives from the heat penetration depth, and thus, indicates a thermal ablation process. For galvanized steel, $B_{F_{t h}}=0.6 \pm 0.08$ indicates a thermally dominated material removal process. That is, laser material removal of galvanized steel is dominated by phase explosion than spallation and vaporization within the context of our work.

The absorbed part of laser energy is lost partly in material removal and partly as residual thermal energy [60]. At long laser pulses, heat penetrates deeper in the material and thermalizes a larger volume. On the contrary, thermal energy from absorbed laser pulse is confined close to the surface for shorter pulse durations. The residual rate of single pulse energy $\eta_{\text {res }}$ after material removal can be calculated from the ablated volume [52,61]. Figure 7 (c) shows the percentage of incident laser energy retained in the sample after irradiating with single laser pulse $(N=1)$ at different pulse durations. Since a larger volume is affected and ablated when processing with longer laser pulses, $\eta_{\text {res }}$ decreases with increasing pulse duration. For fs laser pulses, incident laser energy retained in the sample has been reported 
to be as high as $70 \%$ [60]. This observation is related to the material removal mechanism in laser ablation. The onset of spallation is accompanied by interrupted heat conduction, because the heated surface gets separated from the bulk [12]. Therefore, melting depth drops sharply and resolidification happens faster. On the other hand, the onset of phase explosion facilitates melt expulsion and the redistribution of molten material through recoil pressure from the expanding ablation plume as well as from laser-induced stress relaxation [12]. During phase explosion, continued interaction of the target surface with the ablation plume takes place, which results in the increased duration of the melting and the resolidification cycle, as well as the increased size of the melt zone [12].

Moreover, the ablated plasma in ambient atmosphere leads to residual heating of the sample through thermal conduction [62] and laser-supported absorption waves [60]. The ablated plume forms a plasma due to the high temperature in the material plume [63]. Presence of background gases, such as air, creates a plasma confinement close to target surface. While expanding, this plasma exerts recoil pressure over the melt and expels it towards the edge of the crater as the primary wave. Due to the high pressure gradient within the expanding plume, secondary, or even tertiary molten waves might also be generated [64]. The expanding plasma creates a background pressure drop over the molten surface [64]. If the thickness of melt is sufficient, it might be drawn up by the background pressure drop. This can be observed for $N=1$ at $\tau=30$ ns in Fig. 2. Recoil pressure from the ablated particles increases almost linearly with increasing laser intensity, and consequently, reduces the melt thickness [2]. Liquid phase expulsion depends on the thickness of the melt. As the pulse duration shortens towards picoseconds, the melt depth reduces compared to nanosecond laser pulses. Therefore, the primary wave solidifies rapidly in a splash shape due to hydrodynamic instabilities before reaching the edge of the crater [22]. As a result, no rim forms around the crater for ps pulses (see Fig. 4). In the case of fs pulses, the electron subsystem heats up long before the lattice, which results in an absence of liquid phase during the laser-material interaction. Therefore, the laser pulse is long gone before phase change of the target takes place, resulting in non-thermal ablation [13]. For long laser pulse durations, material removal is dominated phase explosion and/or vaporization at high fluences, whereas material removal is dominated by liquid phase expulsion at low laser fluences (close to but higher than the ablation threshold) [2]. Therefore, at $F_{0}=20 \mathrm{~J} / \mathrm{cm}^{2}$ for single pulse, liquid phase expulsion of thicker melt may be responsible for the maximum 

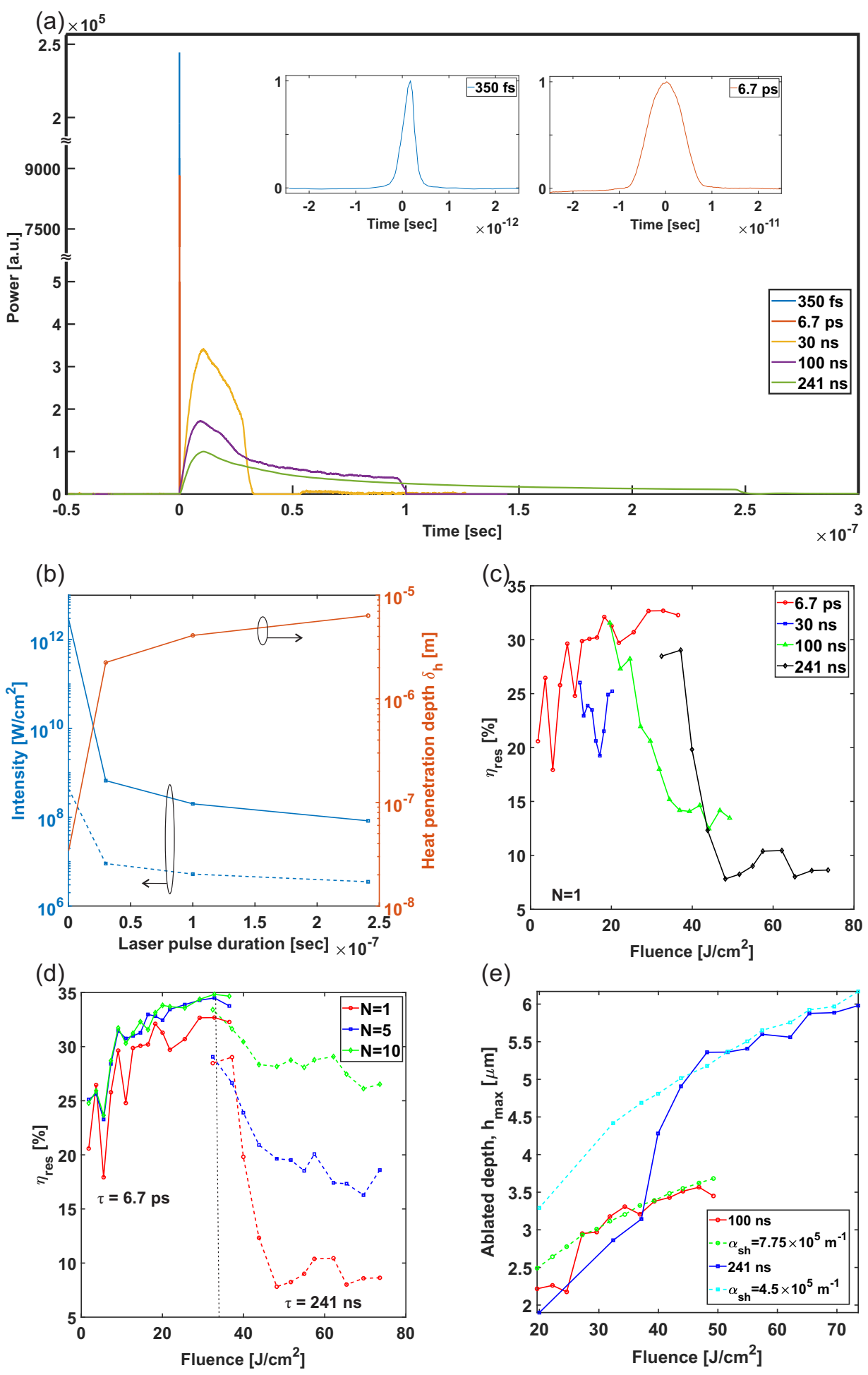

FIG. 7. (a) Measured temporal profile of the laser pulses. Insets show the normalized temporal profiles of femto- and picosecond laser pulses. (b) $I_{0}, I_{\text {evap }}$ and $\delta_{h}$ vs. $\tau_{L}$. (c) $\eta_{\text {res }}$ vs. $F_{0}$ for different pulse durations at $N=1$. (d) $\eta_{\text {res }}$ vs. $F_{0}$ for $N=1,5$ and 10 at $\tau_{L}=6.7$ ps and $241 \mathrm{~ns}$. (e) Experimental and simulated ablated depth as a function of peak fluence for $N=1$ at $\tau_{L}=100$ ns and 241 ns. 
ablated depth at a pulse duration of $100 \mathrm{~ns}$ than $30 \mathrm{~ns}$ (see Fig. 2(c) and (e)). Over multiple laser pulses, the material removal, resulting from both the phase explosion and liquid phase expulsion, maximizes the ablated depth than the material removal resulting from dominant liquid phase expulsion (see Fig. 2(d) and (f)). It appears from Fig. 2 that the ablation efficiency is highest at $\tau_{L}=30 \mathrm{~ns}$, which is contrary to the conclusions drawn in Sec. III B. At a constant fluence, higher ablation efficiency for shorter pulse durations in the nanosecond regime was reported for steel [65] and $\mathrm{Cu}$ [66]. However, material removal at a given laser fluence depends on the material properties like threshold fluence and energy penetration depth for different laser pulse durations. The optimum fluence value for material removal is approximately 7.4 times the threshold fluence [51]. Since threshold fluence scales with pulse duration, the optimum fluence value for material removal increases with increasing pulse duration. That is why, the increase in ablation efficiency with increasing pulse duration is clearly visible in Fig. 3 than Fig. 2.

The initial plasma properties, such as plume temperature, electron density, plasma expansion length and position of the shock front, increase with the intensity of the laser pulse [67]. Plume-splitting into fast and slow components takes place during plasma expansion into low pressured gases, where the fast species expand at vacuum velocity [2]. The 'free' expansion length of the plasma plume is given by $l_{h p}=v_{p} \tau$, where $\tau$ is a time duration (e.g. pulse duration) and $v_{p}=\sqrt{\gamma k_{B} T_{p} / M}$ is the plasma expansion velocity with an adiabatic coefficient of ideal gas $\gamma$, Boltzmann constant $k_{B}$, plasma temperature $T_{p}$ and atomic mass $M$ [68]. Under these crude assumptions, the plasma will expand over longer distance within the laser pulse duration for longer laser pulses. As a consequence, the vapor pressure close to surface will decrease and facilitate higher evaporation rate. Therefore, at a constant intensity, longer pulses will ablate more material (see Fig. 5).

Moreover, the vapor plume density, plasma expansion length, plume velocity and surface temperature increase with increasing pulse duration at a constant intensity [67]. This is because the fluence increases with increasing pulse duration at constant intensity. As the density of the ablated particles increases, the incident laser beam is absorbed and/or reflected by the ablated plasma. Since the wavelengths of the laser sources are in the near infrared regime, absorption of the laser beam within the plasma plume will be dominated 
by the inverse Bremsstrahlung mechanism [2]. Therefore, the reduction of laser energy on the target sample due to plasma shielding will increase with increasing pulse durations at a constant intensity. Lutey modeled material ablation by nanosecond laser pulses using the classical heat conduction equation and temperature dependent material properties [19]. Using a similar model for $\mathrm{Zn}$, the shielding coefficient $\alpha_{s h}$ can be estimated by fitting the simulated depth with the experimental data. In Fig. 7(e), the simulated and experimental ablated depth of the craters are shown for $100 \mathrm{~ns}$ and $241 \mathrm{~ns}$ pulse durations. As can be seen from this figure, $\alpha_{s h}$ is higher for $100 \mathrm{~ns}$ than $241 \mathrm{~ns}$ pulse duration for similar fluence levels. This is because, at a constant fluence, intensity is higher for $100 \mathrm{~ns}$ pulses than 241 ns pulses. Therefore, the plume density is higher at shorter pulse duration, leading to higher plasma shielding. However, the simulated depth at the given $\alpha_{s h}$ deviates significantly at low fluence levels, close to ablation threshold, for both the laser pulse durations. This could be attributed to the surface roughness of the unprocessed surface. Surface roughness affects the laser-material interaction [52], causes plume deflection [69] and influences plasma emission intensity [70]. Therefore, the shielding coefficient appears to be higher at low fluence levels close to ablation threshold, which results in lower ablated depth than the depth predicted by the simulation (see Fig. $7(\mathrm{e})$ ).

For multiple laser pulse processing $(N>1)$, the time between consecutive pulses determines the residual heating of the sample. As a consequence, heat accumulation and particle shielding will affect the ablation efficiency [71]. In Fig. 7(d), $\eta_{\text {res }}$ for laser ablated craters at $6.7 \mathrm{ps}$ laser pulse and at $241 \mathrm{~ns}$ laser pulse is shown for 1, 5 and 10 pulses, impinging $7 \mathrm{~ms}$ after the previous pulse on the same location. For 6.7 ps laser pulses, there lies almost no difference in $\eta_{\text {res }}$ with an increasing number of pulses. On the contrary, the differences in $\eta_{\text {res }}$ with increasing number of pulses is significant for $241 \mathrm{~ns}$ laser pulses. This observation indicates that the sample did not cool down to room temperature within $7 \mathrm{~ms}$ before the next pulse arrives, when processing at 241 ns pulse duration. Therefore, low pulse repetition rates $(<100 \mathrm{~Hz})$ should be used to avoid heat accumulation, when processing with long laser pulses $\left(\tau_{L}<100 \mathrm{~ns}\right)$. 

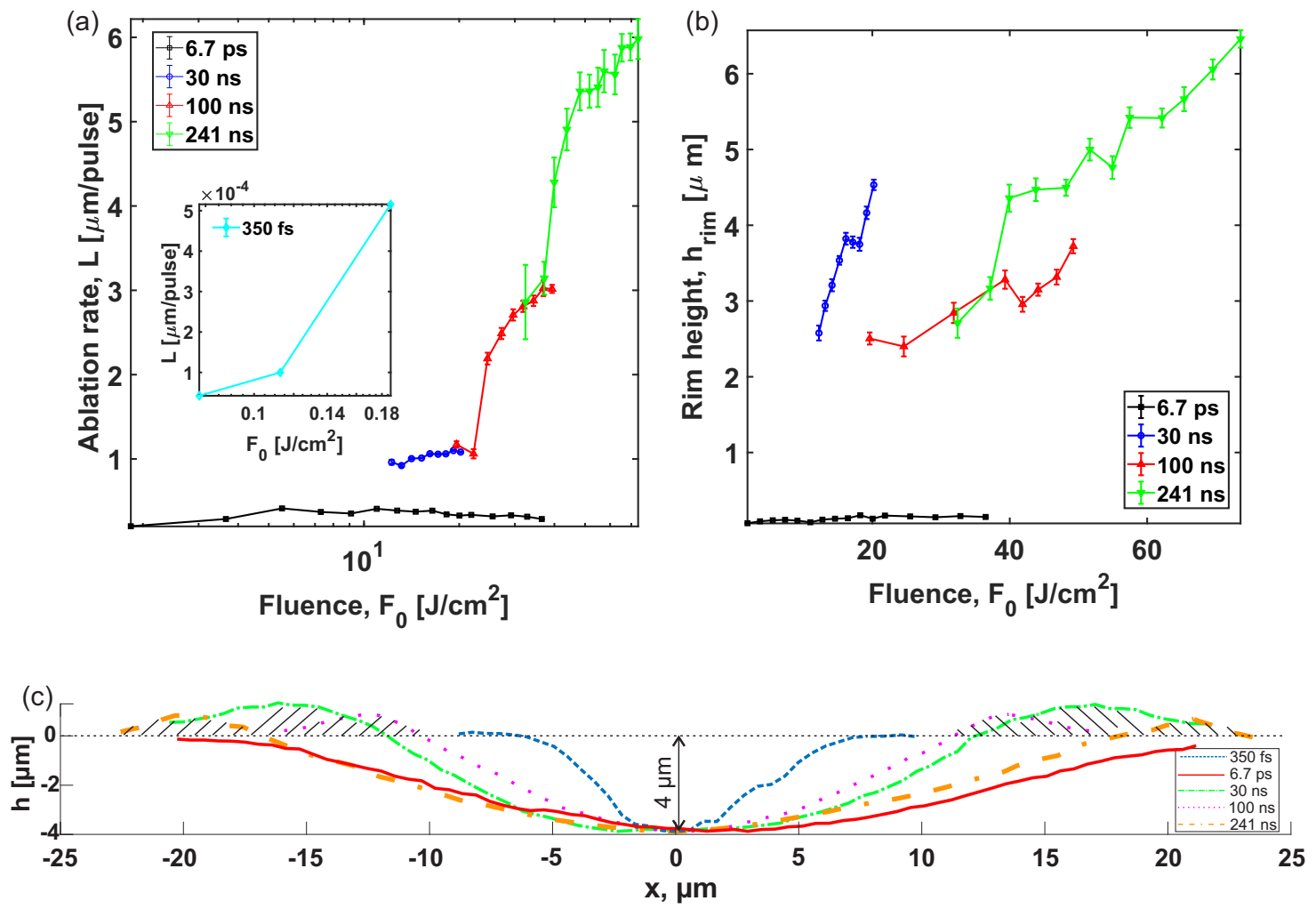

FIG. 8. (a) Ablation rate $L=h_{\max } / N$ of galvanized steel at near infrared wavelengths in air for $h_{\max } \leq 6 \mu \mathrm{m}$ as a function of peak laser fluence at laser pulse durations of $6.7 \mathrm{ps}(N=10), 30$ ns $(N=5), 100 \mathrm{~ns}(N=2)$ and $241 \mathrm{~ns}(N=1)$. Inset shows $L$ for laser pulse duration of $350 \mathrm{fs}$ $\left(N=50 \cdot 10^{3}\right)$. (b) Rim height $h_{\text {rim }}$ around the ablated crater as a function of laser fluence $F_{0}$ at $N=10$ for different laser pulse durations. (c) Cross-sections (obtained from CLSM measurements) of ablated craters with a depth of $4 \mu \mathrm{m}$. The craters were processed at different $F_{0}$ and $N$ at $350 \mathrm{fs}$ $\left(F_{0}=0.2 \mathrm{~J} / \mathrm{cm}^{2}, N=50 \times 10^{3}\right), 6.7 \mathrm{ps}\left(F_{0}=5.5 \mathrm{~J} / \mathrm{cm}^{2}, N=10\right), 30 \mathrm{~ns}\left(F_{0}=13 \mathrm{~J} / \mathrm{cm}^{2}, N=5\right)$, $100 \mathrm{~ns}\left(F_{0}=23 \mathrm{~J} / \mathrm{cm}^{2}, N=5\right)$ and $241 \mathrm{~ns}\left(F_{0}=38 \mathrm{~J} / \mathrm{cm}^{2}, N=1\right)$. The shaded regions indicate the rim. The axes are in equal length scale.

\section{SELECTION OF PULSE DURATION AS A PROCESSING TOOL}

In the context of material processing, (ultra)short-pulsed laser processing results in different depth resolutions and surface morphologies with different laser pulse durations. The lower the ablation rate (ablated depth per pulse), the higher the depth resolution. That is, the number of atomic layers removed per laser pulse largely depends on the pulse duration. Shorter pulse duration results in shorter energy penetration depth, and consequently, lower 
ablation rate per pulse. On the other hand, the ablation efficiency increases with increasing pulse duration. That is, a higher number of atomic layers are removed per pulse for longer pulse durations. Therefore, there lies a trade-off between ablation efficiency and depth resolution. In Fig. 8(a), the average ablation rates of galvanized steel at different pulse durations are plotted against peak fluence. As can be observed, the increment in ablated depth gets steeper as pulse duration increases, although the change of ablating material slows down the ablation rate due to the change in material properties, e.g. melting point, density, thermal diffusivity etc. Within our experimental conditions, only picosecond laser pulses experience a saturation in ablated depth, if the maximum ablated depth reaches the coating substrate interface. On the other hand, saturation in ablated depth, due to the change in ablating material, was not observed when processing with fs and ns laser pulses. As discussed, the depth resolution, i.e. the minimum ablated depth per pulse, is higher for shorter pulse durations than the longer ones.
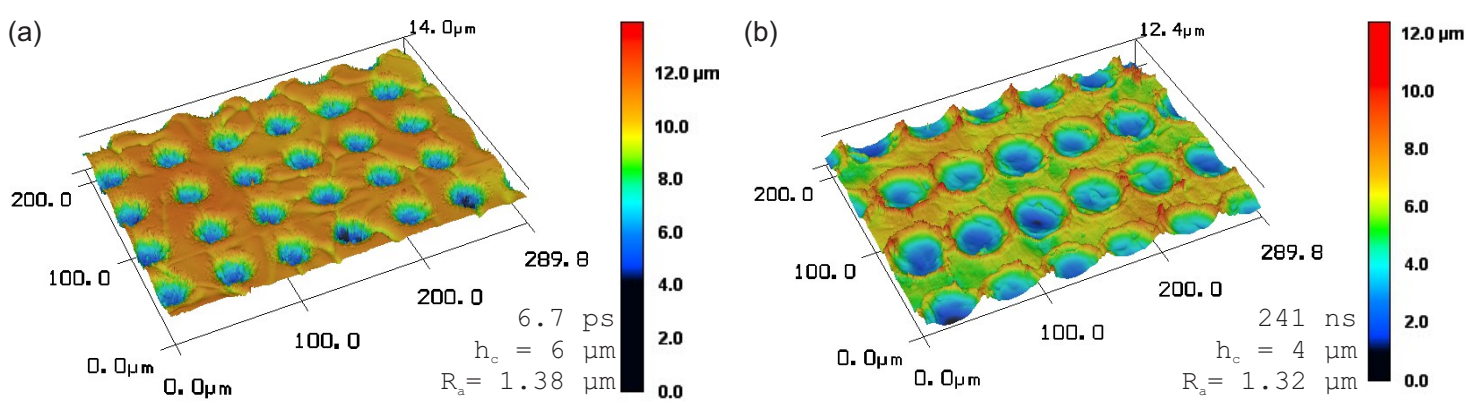

FIG. 9. 3D height profile of galvanized steel surfaces processed for identical surface roughness $\left(R_{a}=1.3 \mu \mathrm{m}\right)$ with (a) $6.7 \mathrm{ps}$ laser pulses at $F_{0}=5 \mathrm{~J} / \mathrm{cm}^{2}, N=20$ resulting in a crater depth $h_{c}$ of $6 \mu \mathrm{m}$ and (b) $241 \mathrm{~ns}$ laser pulses at $F_{0}=40 \mathrm{~J} / \mathrm{cm}^{2}, N=1$ resulting in a crater depth $h_{c}$ of 4 $\mu \mathrm{m}$ and a rim height $h_{\text {rim }}$ of $2 \mu \mathrm{m}$.

As the laser pulse duration increases, the volume of both the recast and the ablated material increases as well. The recast/redeposited material accumulates around the edge of the crater, forming a corona/rim. This is analogous to other "thermal" processes, such as electron beam texturing (EBT) and electrical discharge machining (EDM). Figure 8(b) shows the rim height as a function of peak fluence for different pulse durations at $N=10$. For $\tau<1 \mathrm{~ns}$, the rim height $h_{\text {rim }}$ (and consequently the rim volume) depends more on the increasing number of laser pulses $N$ than increasing peak laser fluence $F_{0}$. In contrast, the 
dependence of $h_{\text {rim }}$ on $F_{0}$ becomes more pronounced for $\tau>1$ ns. In Fig. 8(c), the crosssections of the ablated craters with a depth of $4 \mu \mathrm{m}$ is shown for different pulse durations. The shaded area above $h=0$ represents a cross-section of the rim volume around the crater, which is only visible for ns pulses. For fs and ps pulses, the craters do not show a rim. The rim increases the surface roughness as shown in Fig. 9. Depending on the functionality, rim formation might not necessarily be detrimental. However, the number of laser pulses required to produce craters per unit depth increases with decreasing pulse duration at the cost of higher peak fluence.

\section{CONCLUSION}

The influence of laser pulse duration on the ablation threshold and the ablation efficiency of galvanized steel at near infra-red wavelengths was investigated. The morphological analysis revealed the effect of different physical mechanisms, resulting from different laser pulse durations, on the final shape of the crater. The ablation threshold fluence was found to increase with the laser pulse duration, following a simple power law with an exponent of 0.6. The ablation efficiency also increased in a similar trend as the threshold fluence with increasing pulse duration, but with a smaller exponent value of 0.29 . The increment in ablation efficiency is due to higher energy penetration depth at longer pulse durations, resulting in a relatively higher amount of melting and evaporation of the material. As a consequence, the depth resolution decreases with increasing pulse duration. On the other hand, the volume of both the ablated and recast material increases with laser pulse duration. Also, rim formation around the crater increases for longer pulse durations. Hence, for processing galvanized steel, shorter pulses $(\tau<10 \mathrm{ps})$ are better for precise surface texturing due to higher depth resolution, whereas longer pulse durations $(\tau>1 \mathrm{~ns})$ are better for increased material removal due to higher ablation efficiency.

\section{ACKNOWLEDGMENTS}

The authors would like to acknowledge the financial support of Tata Steel Nederland Technology BV. We would like to thank L. Capuano of University of Twente for his help with the experimental setup and Dr. M. Jalaal, Dr. B. Pathiraj and Dr. ir. W. Ya of the 
University of Twente for their fruitful discussions on this topic.

\section{Appendix A: Equations for calculating timescales}

- The electron relaxation time can be approximated by $\tau_{e-e}=\omega_{p}^{-1}$, where $\omega_{p}$ is plasma frequency [14].

- Electron-phonon coupling time can be calculated as $\tau_{e-p h}==\left(\frac{8}{\pi}\right)^{1 / 4} \sqrt{\frac{A_{e} \cdot T_{m} \cdot C_{l}}{g_{e-p h}^{2}}}$, where $A_{e}, T_{m}, C_{l}$ and $g_{e-p h}$ are the electron specific heat constant, melting point, lattice heat capacity and electron-phonon coupling constant respectively [10].

- The heat conduction happens on a timescale given by $\tau_{\text {heat }}=\frac{L^{2} \rho C_{l}}{K_{l}}$, where $L, \rho$, and $K_{l}$ are the characteristic length scale, density, and lattice thermal conductivity, respectively [22]. $L$ is taken as $40 \mathrm{~nm}$.

- Pulse duration for stress confinement is given by $\tau_{\text {stress }}=\frac{L_{\text {electron }}}{C_{s}}$, where $L_{\text {electron }}=$ $\left(\frac{128}{\pi}\right)^{1 / 8}\left(\frac{K_{l}^{2} \cdot C_{l}}{A_{e} \cdot T_{m} \cdot g_{e-p h}^{2}}\right)^{1 / 4}$ is the electronic diffusion length, and $C_{s}$ is the velocity of sound in the material [12].

- Pulse duration for thermal confinement is given by $\tau_{\text {thermal }}=\frac{\delta_{\text {total }}}{D}$, where $D=\frac{K_{l}}{C_{l}}$ is thermal diffusivity and $\delta_{\text {total }}$ is the total (maximum) penetration depth of photons $\delta_{\text {opt }}$ and electrons $\delta_{b a l}[12]$.

- Visco-capillary timescale can be calculated as $\tau_{v i s-c a p}=\frac{L \mu}{\sigma}$ where $\mu$, and $\sigma$ are viscosity, and surface tension, respectively [22]. $L$ is taken as $40 \mathrm{~nm}$.

- Flow timescale can be calculated as $\tau_{f l o w}=\frac{\mu L^{2}}{P_{\text {ave }} h_{\text {melt }}}$, where $P_{\text {ave }}$ and $h_{\text {melt }}$ are the plasma pressure and the melt thickness, respectively [22]. $L$ and $h_{m e l t}$ are taken as 40 $\mathrm{nm}$ and $1 \mu \mathrm{m}$ respectively.

- Critical pulse duration is given by $\tau_{c}=\left(\frac{8}{\pi}\right)^{1 / 4} \sqrt{\frac{C_{l}^{3}}{A_{e} \cdot T_{m} \cdot g_{e-p h}^{2}}}[11]$.

- Duration of the ablation process can be estimated as $\tau_{\text {ablation }}=\frac{L^{*}}{\sqrt{\Sigma_{\text {vap }}}}$, where $L^{*}$ is taken as $1 \mu \mathrm{m}[20]$.

- Time for conventional heat diffusion is calculated as $t_{\text {heat }}=\frac{2 . c . \lambda_{\text {laser }}}{\omega_{p} . D}$, where $c$ and $\lambda_{\text {laser }}$ are the speed of light and laser wavelength respectively [14]. 


\section{Appendix B: Material properties}

TABLE II. Material Properties of Zinc

\begin{tabular}{lllll}
\hline \hline Parameter & Symbol & Value & Unit & Ref. \\
\hline Plasma frequency & $\omega_{p}$ & 10.1 & $\mathrm{eV}$ & {$[72]$} \\
Lattice thermal conductivity & $K_{l}$ & 116 & $\mathrm{~W} \cdot \mathrm{m}^{-1} \cdot \mathrm{K}^{-1}$ & {$[73]$} \\
Lattice heat capacity & $C_{l}$ & 2.76 & $\mathrm{~J} \cdot \mathrm{cm}^{-3} \cdot \mathrm{K}^{-1}$ & {$[74]$} \\
Electron specific heat constant & $A_{e}$ & 68.382 & $\mathrm{~J} \cdot \mathrm{m}^{-3} \cdot \mathrm{K}^{-1}$ & {$[75]$} \\
Melting point & $T_{m}$ & 692.68 & $\mathrm{~K}$ & {$[76]$} \\
Electron-phonon coupling coefficient & $g_{e-p h}$ & $6.4 \times 10^{16}$ & $\mathrm{~W} \cdot \mathrm{m}^{-3} \cdot \mathrm{K}^{-1}$ & {$[77]$} \\
Density & $\rho$ & 7140 & $\mathrm{~kg} \cdot \mathrm{m}^{-3}$ & {$[76]$} \\
Latent heat of vaporization & $\Omega_{v a p}$ & $1748 \times 10^{3}$ & $\mathrm{~J} \cdot \mathrm{kg}$ & {$[73]$} \\
Velocity of sound at 300K, rolled & $C_{s}$ & 3850 & $\mathrm{~m} / \mathrm{s}$ & {$[78]$} \\
Viscosity at 700K & $\mu$ & $3.737 \times 10^{-3}$ & $\mathrm{~Pa} \cdot \mathrm{s}$ & {$[79]$} \\
Surface tension at 700K & $\sigma$ & 0.83 & $\mathrm{~N} / \mathrm{m}$ & {$[80]$} \\
\hline \hline
\end{tabular}

[1] M. Mozetič, Materials 12 (2019), 10.3390/ma12030441.

[2] D. Bäuerle, Laser Processing and Chemistry (Springer Berlin Heidelberg, 2011).

[3] B. Jaeggi, B. Neuenschwander, M. Schmid, M. Muralt, J. Zuercher, and U. Hunziker, Physics Procedia 12, 164 (2011).

[4] B. Neuenschwander, B. Jaeggi, and M. Schmid, Physics Procedia 41, 794 (2013).

[5] B. Neuenschwander, B. Jaeggi, M. Schmid, V. Rouffiange, and P.-E. Martin, in SPIE LASE (International Society for Optics and Photonics, 2012) pp. 824307-824307.

[6] A. Semerok, C. Chaléard, V. Detalle, J.-L. Lacour, P. Mauchien, P. Meynadier, C. Nouvellon, B. Sallé, P. Palianov, M. Perdrix, et al., Applied Surface Science 138, 311 (1999).

[7] P. Lorazo, L. J. Lewis, and M. Meunier, Physical Review B 73, 134108 (2006).

[8] R. E. Russo, X. L. Mao, J. H. Yoo, and J. J. Gonzalez, "Laser ablation," in Laser-induced breakdown spectroscopy, edited by J. P. Singh and S. N. Thakur (Elsevier, 2007) Chap. 3, pp. 
49-82.

[9] J. Hohlfeld, S.-S. Wellershoff, J. Güdde, U. Conrad, V. Jähnke, and E. Matthias, Chemical Physics 251, 237 (2000).

[10] S.-S. Wellershoff, J. Hohlfeld, J. Güdde, and E. Matthias, Applied Physics A 69, S99 (1999).

[11] P. Corkum, F. Brunel, N. Sherman, and T. Srinivasan-Rao, Physical review letters 61, 2886 (1988).

[12] L. V. Zhigilei, Z. Lin, and D. S. Ivanov, The Journal of Physical Chemistry C 113, 11892 (2009).

[13] B. Rethfeld, D. S. Ivanov, M. E. Garcia, and S. I. Anisimov, Journal of Physics D: Applied Physics 50, 193001 (2017).

[14] E. G. Gamaly, A. V. Rode, B. Luther-Davies, and V. T. Tikhonchuk, Physics of plasmas 9, 949 (2002)

[15] J. Hohlfeld, J. Müller, S.-S. Wellershoff, and E. Matthias, Applied Physics B 64, 387 (1997).

[16] M. Agranat, S. Ashitkov, V. Fortov, A. Kirillin, A. Kostanovskii, S. Anisimov, and P. Kondratenko, Applied Physics A: Materials Science \& Processing 69, 637 (1999).

[17] J. C. Miller and R. F. Haglund, Laser ablation and desorption, EXPERIMENTAL METHODS IN THE PHYSICAL SCIENCES, Vol. 30 (Elsevier, 1998).

[18] L. Zhigilei and B. J. Garrison, Applied Physics A 69, S75 (1999).

[19] A. H. Lutey, Journal of Applied Physics 114, 083108 (2013).

[20] C. Momma, B. N. Chichkov, S. Nolte, F. von Alvensleben, A. Tünnermann, H. Welling, and B. Wellegehausen, Optics communications 129, 134 (1996).

[21] F. Spaepen, High Temperature Materials and Processes 7, 91 (1986).

[22] H. Mustafa, M. Jalaal, W. Ya, N. Ur Rahman, D. T. A. Matthews, and G. R. B. E. Römer, Journal of Laser Micro/Nanoengineering 13, 178 (2018).

[23] R. Fang, A. Vorobyev, and C. Guo, Light: Science \& Applications 6, e16256 (2017).

[24] H. Mustafa, R. Pohl, T. C. Bor, B. Pathiraj, D. T. A. Matthews, and G. R. B. E. Römer, Optics Express 26, 18664 (2018).

[25] M. Akram, S. Bashir, A. Hayat, K. Mahmood, R. Ahmad, and M. Khaleeq-U-Rahaman, Laser and Particle Beams 32, 119 (2014).

[26] I. Daigo, S. Osako, Y. Adachi, and Y. Matsuno, Resources, Conservation and Recycling 82, $35(2014)$. 
[27] G. Meylan and B. K. Reck, Resources, Conservation and Recycling 123, 1 (2017).

[28] L. M. Cabalín, A. González, V. Lazic, and J. Laserna, Applied spectroscopy 65, 797 (2011).

[29] C. Garcia, M. Corral, J. M. Vadillo, and J. Laserna, Applied Spectroscopy 54, 1027 (2000).

[30] H. Balzer, M. Hoehne, V. Sturm, and R. Noll, Spectrochimica Acta Part B: Atomic Spectroscopy 60, $1172(2005)$.

[31] T. Canel, P. Demir, E. Kacar, B. G. Oztoprak, E. Akman, M. Gunes, and A. Demir, Optics \& Laser Technology 54, 257 (2013).

[32] A. Hrdlička, V. Otruba, K. Novotnỳ, D. Günther, and V. Kanickỳ, Spectrochimica Acta Part B: Atomic Spectroscopy 60, 307 (2005).

[33] M. P. Mateo, C. C. Garcia, and R. Hergenröder, Analytical chemistry 79, 4908 (2007).

[34] J. M. Vadillo, C. C. García, S. Palanco, and J. J. Laserna, Journal of Analytical Atomic Spectrometry 13, 793 (1998).

[35] J. M. Vadillo and J. Laserna, Journal of Analytical Atomic Spectrometry 12, 859 (1997).

[36] A. Marder, Progress in materials science 45, 191 (2000).

[37] S. Shibli, B. Meena, and R. Remya, Surface and Coatings Technology 262, 210 (2015).

[38] C. Li, R. Ma, A. Du, Y. Fan, X. Zhao, and X. Cao, Metals 8, 687 (2018).

[39] F. J. M. Ruiz-Cabello, A. Amirfazli, M. Cabrerizo-Vílchez, and M. Rodríguez-Valverde, RSC Advances 6, 71970 (2016).

[40] O. Pawelski, W. Rasp, W. Zwick, H.-J. Nettelbeck, and K. Steinhoff, Journal of materials processing technology 45, 215 (1994).

[41] A. Singh, D. S. Patel, J. Ramkumar, and K. Balani, The International Journal of Advanced Manufacturing Technology 100, 1253 (2019).

[42] X. Wang, A. Kuchmizhak, D. Storozhenko, S. Makarov, and S. Juodkazis, ACS Applied Materials \& Interfaces 10, 1422 (2018), pMID: 29250954, https://doi.org/10.1021/acsami.7b16339.

[43] R. Kromer, S. Costil, C. Verdy, S. Gojon, and H. Liao, Surface and Coatings Technology 352, 642 (2018).

[44] D. Cole, The splashing morphology of liquid-liquid impacts, Ph.D. thesis, James Cook University (2007).

[45] J. Picard, J.-B. Sirven, J.-L. Lacour, O. Musset, D. Cardona, J.-C. Hubinois, and P. Mauchien, Spectrochimica Acta Part B: Atomic Spectroscopy 101, 164 (2014).

[46] M. Hashida, A. F. Semerok, O. Gobert, G. Petite, and J.-F. Wagner, in Nonresonant Laser- 
Matter Interaction (NLMI-10) (International Society for Optics and Photonics, 2001) pp. $178-185$.

[47] J. Liu, Optics letters 7, 196 (1982).

[48] P. Mannion, J. Magee, E. Coyne, G. O'connor, and T. Glynn, Applied Surface Science 233, 275 (2004).

[49] S. Preuss, A. Demchuk, and M. Stuke, Applied Physics A: Materials Science \& Processing 61, 33 (1995).

[50] G. Račiukaitis, M. Brikas, P. Gecys, and M. Gedvilas, in High-Power Laser Ablation 2008 (International Society for Optics and Photonics, 2008) pp. 70052L-70052L.

[51] G. Račiukaitis, M. Brikas, P. Gecys, B. Voisiat, M. Gedvilas, et al., JLMN Journal of Laser Micro/Nanoengineering 4, 186 (2009).

[52] H. Mustafa, M. Mezera, D. Matthews, and G. Römer, Applied Surface Science 488, 10 (2019).

[53] Y. Jee, M. F. Becker, and R. M. Walser, JOSA B 5, 648 (1988).

[54] M. Hashida, A. Semerok, O. Gobert, G. Petite, Y. Izawa, et al., Applied surface science 197, $862(2002)$.

[55] W. Kautek, J. Krüger, M. Lenzner, S. Sartania, C. Spielmann, and F. Krausz, Applied physics letters 69, 3146 (1996).

[56] D. Giguère, G. Olivié, F. Vidal, S. Toetsch, G. Girard, T. Ozaki, J.-C. Kieffer, O. Nada, and I. Brunette, JOSA A 24, 1562 (2007).

[57] B. Neuenschwander, G. F. Bucher, G. Hennig, C. Nussbaum, B. Joss, M. Muralt, S. Zehnder, U. W. Hunziker, and P. Schütz, International Congress on Applications of Lasers \& ElectroOptics 2010, 707 (2010).

[58] X. Zeng, X. Mao, R. Greif, and R. Russo, Applied Physics A 80, 237 (2005).

[59] P. Pronko, S. Dutta, D. Du, and R. Singh, Journal of Applied Physics 78, 6233 (1995).

[60] A. Vorobyev, V. Kuzmichev, N. Kokody, P. Kohns, J. Dai, and C. Guo, Applied Physics A 82, 357 (2006).

[61] B. Wu, P. Liu, X. Wang, F. Zhang, L. Deng, J. Duan, and X. Zeng, Optics \& Laser Technology 101, $11(2018)$.

[62] S. Laville, F. Vidal, T. Johnston, O. Barthélemy, M. Chaker, B. Le Drogoff, J. Margot, and M. Sabsabi, Physical review E 66, 066415 (2002).

[63] A. Bogaerts, Z. Chen, R. Gijbels, and A. Vertes, Spectrochimica Acta Part B: Atomic Spec- 
troscopy 58, 1867 (2003).

[64] A. Otto, H. Koch, R. G. Vázquez, Z. Lin, and B. Hainsey, Physics Procedia 56, 1315 (2014).

[65] G. Dumitru, V. Romano, and H. Weber, Applied Physics A 79, 1225 (2004).

[66] A. Semerok, B. Salle, J.-F. Wagner, and G. Petite, Laser and Particle Beams 20, 67 (2002).

[67] A. Bogaerts and Z. Chen, Spectrochimica Acta Part B: Atomic Spectroscopy 60, 1280 (2005).

[68] M. Stafe and C. Negutu, Plasma Chemistry and Plasma Processing 32, 643 (2012).

[69] A. Perrone, A. Zocco, L. Cultrera, and D. Guido, Applied surface science 197, 251 (2002).

[70] I. Lopez-Quintas, V. Pinon, M. Mateo, and G. Nicolas, Applied surface science 258, 9432 (2012).

[71] A. Ancona, D. Nodop, J. Limpert, S. Nolte, and A. Tünnermann, Applied Physics A 94, 19 (2009).

[72] L. Mosteller Jr and F. Wooten, Physical Review 171, 743 (1968).

[73] L. Cabalin and J. Laserna, Spectrochimica Acta Part B: Atomic Spectroscopy 53, 723 (1998).

[74] D. R. Lide, CRC Handbook of Chemistry and Physics: A Ready-reference Book of Chemical and Physical Data: 1993-1994 (CRC press, 1993).

[75] S. W. Van Sciver, in Helium Cryogenics (Springer, 2012) pp. 17-58.

[76] F. C. Porter, Zinc handbook: Properties, Processing, and Use in design (CRC Press, 1991).

[77] M. Hase, K. Ishioka, J. Demsar, K. Ushida, and M. Kitajima, Physical review B 71, 184301 (2005).

[78] D. R. Lide, CRC Handbook of Chemistry and Physics, 84th ed., Vol. Section 14, Geophysics, Astronomy, and Acoustics; Speed of Sound in Various Media (CRC press, Boca Raton, Florida, 2003).

[79] M. J. Assael, I. J. Armyra, J. Brillo, S. V. Stankus, J. Wu, and W. A. Wakeham, Journal of Physical and Chemical Reference Data 41, 033101 (2012).

[80] W. Falke, A. Schwaneke, and R. Nash, Metallurgical Transactions B 8, 301 (1977). 
Pulse duration

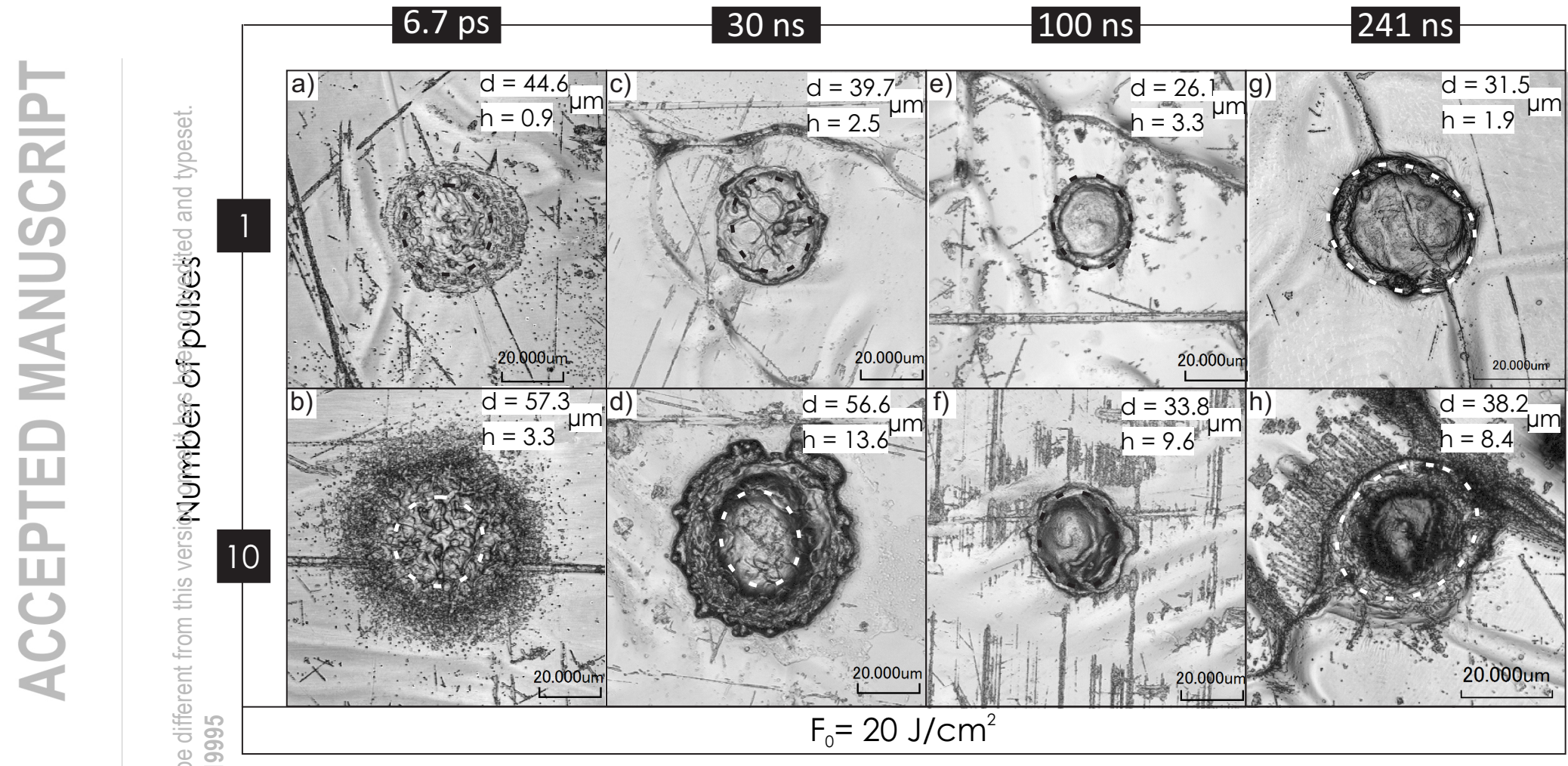



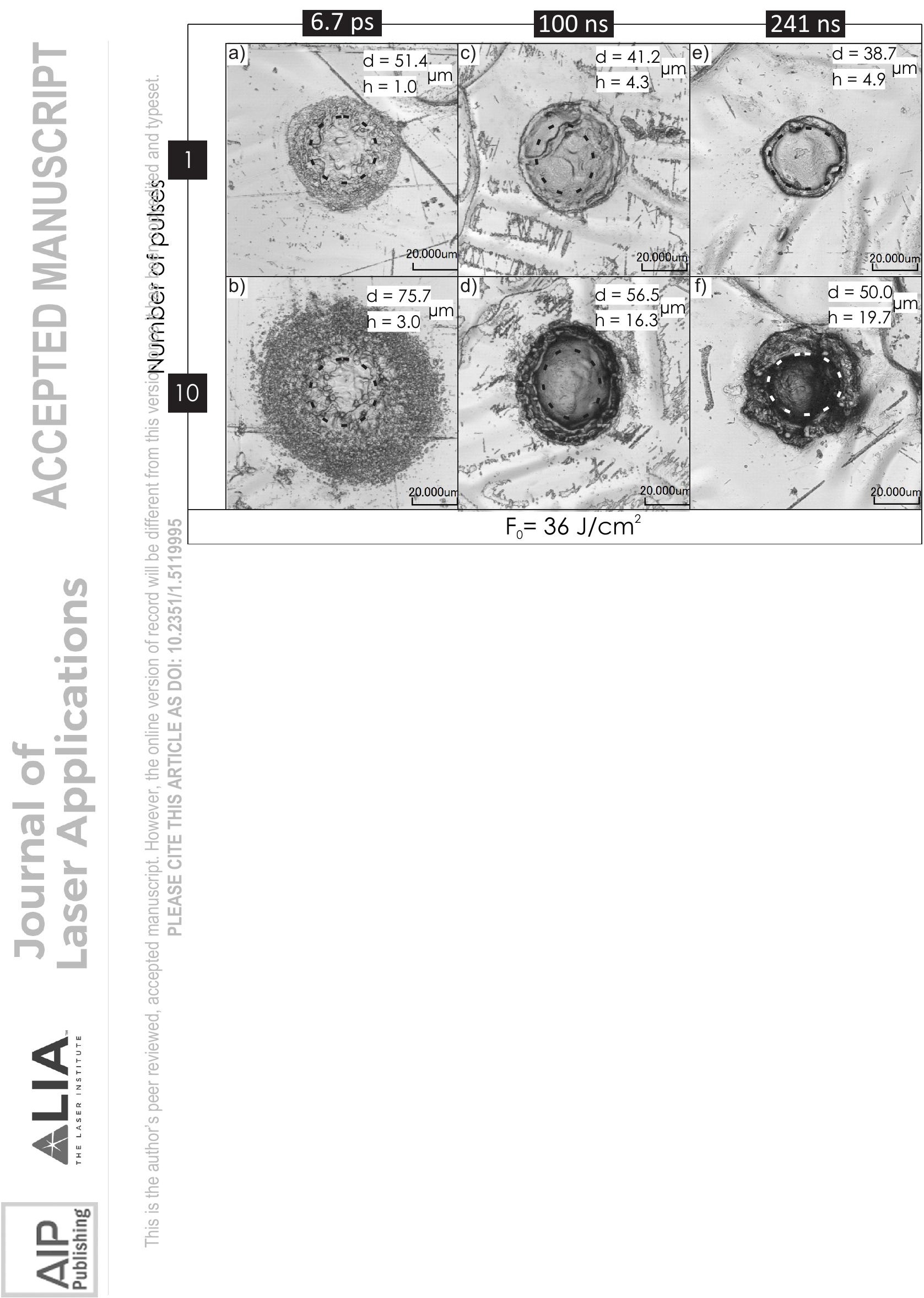


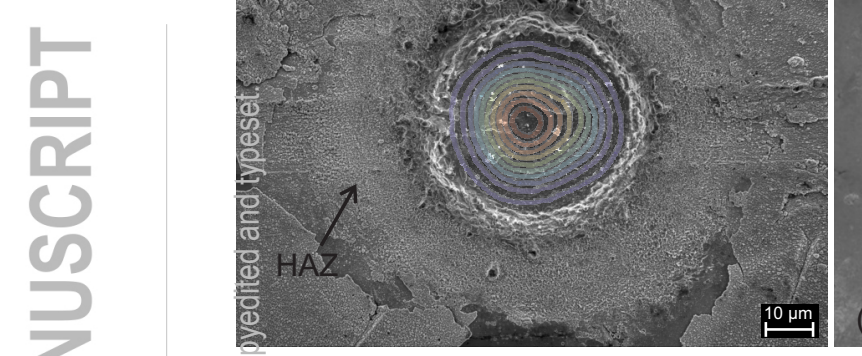

$\stackrel{10 \mu \mathrm{m}}{\longmapsto}$ a)

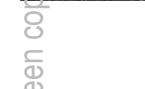

b)

$\mathrm{h}_{\text {rim }}=$ 


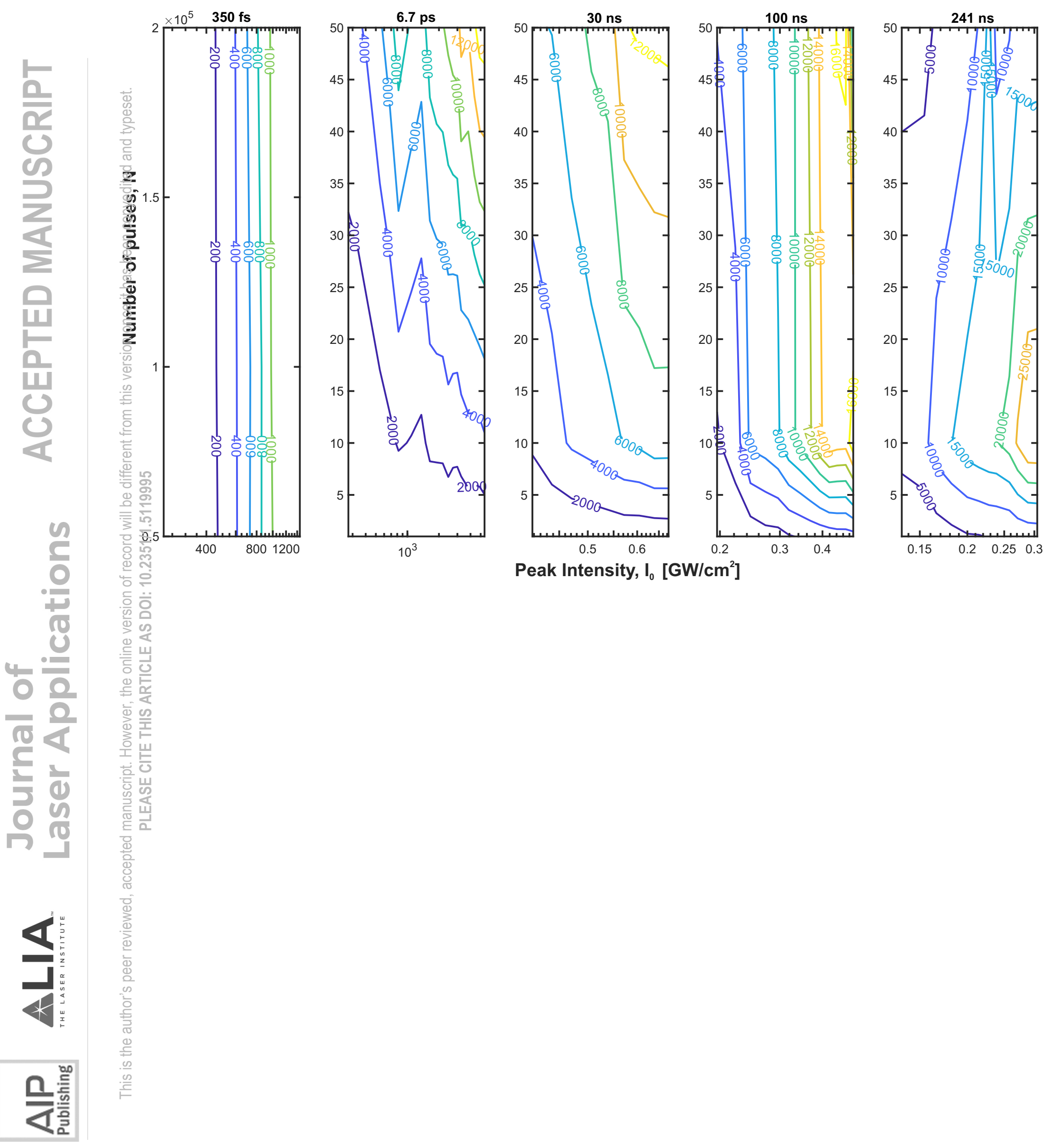


(a)
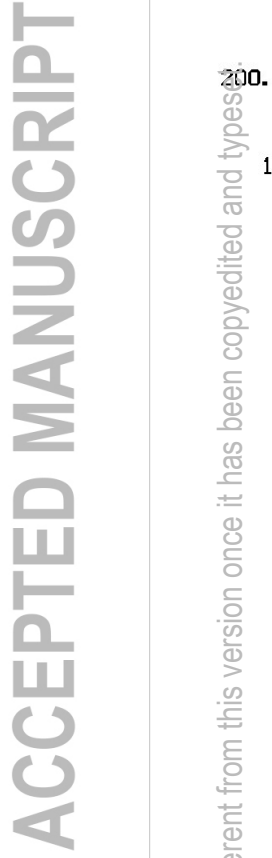

100. 0

D. $\square \mu \mathrm{m}$

0. $0 \mu \mathrm{m}$ (b)

$12.0 \mu \mathrm{m}$

$12.0 \mu$
10.0
8.0
6.0
4.0
2.0
0.0

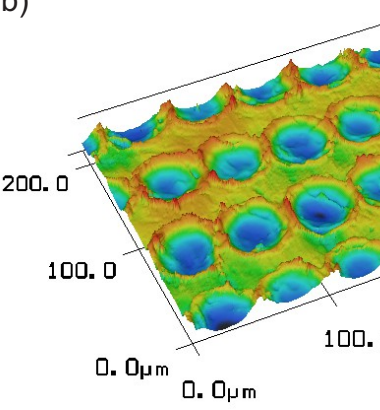

200.0

289. 8

4.0

00. 0

200.0

$6.7 \mathrm{ps}$
$\mathrm{h}_{\mathrm{c}}=6 \mathrm{\mu m}$

$\mathrm{R}_{\mathrm{a}}=1.38 \mu \mathrm{m} \mathbf{0 . 0}_{\mathbf{0}}$

(4)

( 0

$\begin{array}{ll}0 & 0 \\ 10 & 4 \\ 5 & 1 \\ 0 & 1 \\ 1 & 1\end{array}$

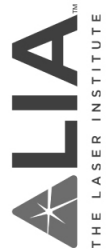

足总 